\title{
Radiographic and Clinical Features of Children With Coronavirus Disease (COVID-19) Pneumonia
}

\author{
Bo LI ${ }^{1}$, Jie Shen ${ }^{2}$, LIANG Li ${ }^{1}$ ANd ChENGXIN Yu ${ }^{1}$ \\ From the Departments of Radiology, ${ }^{1}$ The First College of Clinical Medical Science of China, Three Gorges University and Yichang \\ Central People's Hospital, Yichang; and ${ }^{2}$ The Affiliated Jiujiang Hospital of Nanchang University and Jiu Jiang No.1 People's and \\ Water of Life Hospital, Jiangxi, China.
}

Correspondence to:Dr Chengxin $\mathrm{Yu}$, Department of Radiology, The First College of Clinical Medical Science of China,Three Gorges University and Yichang Central People's Hospital, 183, Yiling Road, Yichang 443003, HuBei Province,China.ycyucx@126.com Submitted: March 22, 2020;

Initial review: March 24, 2020, Accepted: April 4, 2020.
Objective: The purpose of this study was to investigate chest computed tomography (CT) findings in children with coronavirus disease-19 (COVID-19) pneumonia in our hospital. Methods: This study included 22 pediatric patients with confirmed COVID-19 from January to March, 2020. The chest CT images and clinical data were reviewed. Results: The most prevalent presenting symptoms were fever $(64 \%)$ and cough $(59 \%)$, and a mildly elevated mean (SD) C-reactive protein (CRP) level of 11.22(11.06) and erythrocyte sedimentation rateof 18.8(15.17) were detected. The major CT abnormalities observed were mixed ground-glass opacity and consolidation lesions (36\%), consolidations (32\%), and groundglass opacities (14\%). Peripheral distribution (45\%) of lung lesions was predominant. Most of the lesions were multilobar(68\%), with an average of three lung segments involved. Conclusion: Children with COVID-19 had relatively milder symptoms and less severe lung inflammation than adults. Chest CT plays an important role in the management of children with COVID-19 pneumonia.

Keywords: Diagnosis, Evaluation, SARS-CoV-2, Management.

Published online: April 7, 2020; PII: S097475591600156

$\mathrm{T}$ he 2019 novel coronavirus (COVID-19) pneumonia, reported in Wuhan (Hubei Province, China) since late 2019, has garnered intense attention worldwide [1]. The World Health Organization has declared this outbreak as a pandemic. While COVID-19 is usually common in middleaged or elderly people, the incidence of COVID-19 is rare in children, and children have mild clinical symptoms [2]. Chest computed tomography (CT) can identify infected lesions, indicating viral pneumonia, which plays an irreplaceable role in the screening of COVID-19. There are only limited data available regarding the typical chest CT imaging findings of COVID-19 in children [2]. In this study, we retrospectively evaluated radiographic features of chest CT and clinical features in children with confirmed COVID-19.

\section{METHODS}

This study was approved by the Medical Research Ethics Committee of our institution. The requirement for patients' informed consent was waived due to the retrospective nature of the study. From January 16, 2020 to March14, 2020, a search of the electronic system and Picture archiving and communication system (PACS) was performed in our department. All pediatric patients with suspected/proven COVID-19 were being routinely subjected to CT chest at our center. The inclusion criteria were: ( $i$ ) epidemiological history: either travel/residence history in Wuhan or exposure history to patients with fever from Wuhan suffering from respiratory symptoms within 14 days before the onset of illness; and (ii) laboratory diagnosis: positive detection of COVID-19 in throat swabs or lower respiratory tract by real-time fluorescence polymerase chain reaction (Shanghai ZJ Bio-Tech Co, Ltd, Shanghai, China).

The following clinical data of the patients were collected and assessed: sex, age, pharyngeal discomfort, cough, expectoration, chest congestion, myalgia and abdominal pain or diarrhea. Information regarding the physical examination at admission was evaluated, including the heart rate, body temperature, respiratory rate and blood pressure. Moreover, the laboratory data were also assessed, including total and differential leukocyte, erythrocyte sedimentation rate (ESR), procalcitonin, and C-reactive protein (CRP) levels.

Imaging technique: All patients completed non-contrast chest CT scans in a separate examination room while the 
CT technician utilized secondary protection. Chest CT images were obtained using a 16-row multi-detector CT scanner (Siemens Somatom Sensation; Siemens, Erlangen, Germany). The CT examination parameters were as follows: $120 \mathrm{kVp}, 140 \mathrm{~mA}, 5 \mathrm{~mm}$ collimation, $1.35: 1$ pitch, a pulmonary kernel (B70f) and a mediastinal kernel (B30f), reconstruction slice thickness of $1.0 \mathrm{~mm}$, and high spatial resolution algorithm. All the patients over the age of three years were scanned in a supine position while holding their breath at full inspiration, while children under the age of three completed examinations while asleep (were not required to hold their breath).

All chest CT scans were reviewed independently by two senior radiologists, while they were blinded to the name and clinical data of the patients. The two radiologists reached a consensus about the lung abnormalities, and an agreement was reached by discus-sion if the conclusions were different. All CT images were viewed on both lung window (width, $1500 \mathrm{HU}$; level, $500 \mathrm{HU}$ ) and mediastinal window (width, $350 \mathrm{HU}$; level, $40 \mathrm{HU}$ ) settings. The major CT dimensions, including the presence of ground-glass opacities, 'crazy-paving sign', consolidation, and mixed ground-glass opacities and consolidation lesions, were fully evaluated. The detailed definitions of the above features were as per a previous publication $[3,4]$. The distribution of lung abnormalities was recorded as predominantly sub-pleural (involving mainly the peripheral one-third of the lung), central (involving mainly the central one-third of the lung), mixed (involving both sub-pleural and central regions), and diffuse (continuous involvement without respect to lung segments) according to a similar report [5]. The scattering patterns of lesions (focal, multifocal and diffuse) were also classified. The number of bilateral lung segments affected by pneumonia was recorded simultaneously.

Statistical analyses: All statistical analyses were conducted using Statistical Package for Social Sciences software version 18.0 (SPSS Inc., Chicago, IL, USA).

\section{RESULTS}

Records of 22 patients (12 males) were included in this study. The mean (SD) age was 8 (6) years. The most prevalent presenting symptoms were fever (14 cases, $64 \%$ ) and cough (13 cases, 59\%). Two patients had no clinical symptoms or chest CT abnormalities; however, they were in close contact with confirmed cases and had positive results on a COVID-19 nucleic acid test. Chest CT scans were obtained at mean (SD) 3 (3) days (range: 011) after the onset of symptoms. Laboratory investigations showed that the most frequent abnormalities were mildly elevated CRP [mean (SD) $=11.2(11.6)]$ and ESR values $[$ mean $(\mathrm{SD})=18.8(15.17)]($ Web Table $\mathbf{I})$.
Most patients showed bilateral lung involvement (15, 68\%) (Table I). Of the spatial distribution of all lesions, the right lower lobe $(9,41 \%)$ was most commonly involved. The average number of infected lung segments was three (range: $0-15$ ) for all patients, with less than three lung segments involved in 55\% patients. The major CT abnormalities observed were mixed ground glass opacities and consolidation lesions $(8,36 \%)$, or consolidations alone (7, 32\%) (Fig.1). In addition, two children (9\%) showed a 'crazy-paving sign' characterized by reticular interlobular septal thickening within patchy ground glass opacities [6]. Multifocal lesions (15 cases, $68 \%$ ) were most common, and patients had lymph node enlargement.

\section{DISCUSSION}

The novel coronavirus related to the MERS and SARS coronaviruses $[7,8]$ has now spread to become a pandemic, with wide-ranging effects. We found that typical clinical symptoms were similar to those of other types of coronavirus infections, such as SARS and MERS [9-11]. CT chest findings were noted to be characteristic and have been detailed.

Table I Computed Tomography Chest findings in Pediatric Patients with COVID-19 $(N=22)$

\begin{tabular}{lr}
\hline Finding & No. $(\%)$ \\
\hline Ground glass opacities & $3(14)$ \\
Crazy-paving sign & $2(9)$ \\
Consolidation & $7(32)$ \\
Ground glass opacities and consolidation & $8(36)$ \\
Lung region distribution & \\
Unilateral & $5(23)$ \\
Bilateral & $15(68)$ \\
Subpleural & $10(45)$ \\
Central & $1(5)$ \\
Mixed & $9(41)$ \\
Lung lobe involved & \\
Right upper lobe & $2(9)$ \\
Right lower lobe & $9(41)$ \\
Left upper lobe & $3(14)$ \\
Left upper lobe & $6(27)$ \\
${ }^{*}$ Lung segments involved & $3(3)$ \\
Distribution & \\
Focal & \\
Multifocal & $3(14)$ \\
Diffuse & $15(68)$ \\
Data represented as no. (\%) or ${ }^{*}$ mean (SD). Two patients & had no \\
chest CT abnormalities. & $2(9)$ \\
\hline
\end{tabular}




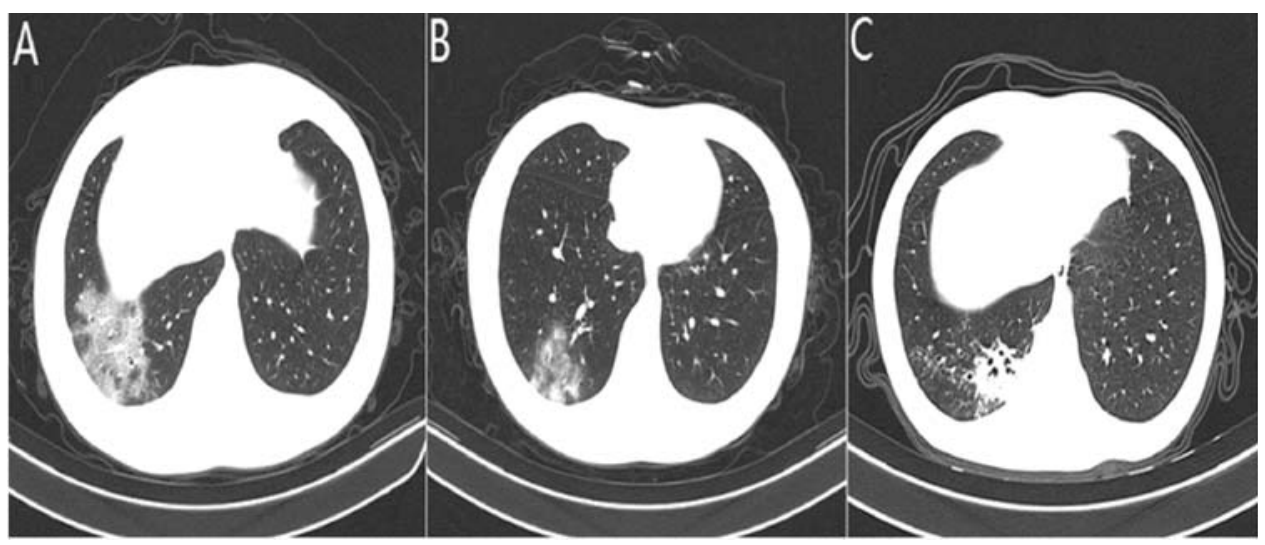

Fig. 1 Computed tomography findings in children with coronavirus-19 (COVID-19) pneumonia: (a) ground glass opacities; (b) consolidation; and (c) ground glass opacities and consolidation.

There is usually a certain time interval between the onset of symptoms and hospitalization of COVID-19 patients. In our study, the time interval between the first chest $\mathrm{CT}$ examination and the onset was 0 to 11 days. An initial negative result of chest $\mathrm{CT}$ examination may occur ( 2 cases, 9\%), but lung abnormalities could be discovered among most of the patients.Most of the patients had mild symptoms as well as temperature elevation, but their lung manifestations were relatively obvious. In contrast tothose in bacterial pneumonia [4], lung lesions showed similar imaging features to other viral pneumonias, mainly ground glass opacities, extensive interlobular septal thickening and patchy consolidation. In the early stage, COVID-19 reflects mainly interstitial lung damage, such as thickening of the interlobular septa and the presence of ground glass opacities. Alveolar edema, exudation and bleeding can be manifested in different degrees of ground glass opacities on CT images, as inflammation involves the alveoli.In more than one-third of cases, abnormal lung manifestations were presented as a mixture of GGOs and consolidations, implying a rapid progression of pneumonia, which is probably due to a lower immune response in children than in adults. In severe cases, owing to the collapse of alveoli or massive infiltration of inflammatory cells, lung parenchymal injury may occur, presentingas lung consolidation. Although more detailed pathological changes of COVID-19 need to be further studied, research has shown that angiotensin-converting enzyme 2 (ACE2) is an important receptor of the SARSCoV-2 surface spike protein domain, which is similar to the case for SARS-CoV. Given that it is highly contagious, its affinity may be greater than that of SARS-CoV [12]. Human ACE2 receptors are abundantly expressed in type II alveolar epithelial cells. The outer bands of the lungs and the sub-pleural space are dense areas where terminal bronchi are dilated to form alveolar ducts, which can also explain the characteristics of lesion distribution from an anatomical perspective. In addition, thickened small blood vessel shadows and faint shadows surrounding the nodules are also characteristic in some reports.

Globally, similar outbreaks of respiratory diseases have also been observed for SARS and MERS, and those causative pathogens belong to the beta-coronavirus family [13]. There is a certain similarity of chest CT imaging features between SARS and COVID-19 [14]. For instance, both GGOs and consolidations are dominant and concentrate mainly in bilateral sub-pleural areas; otherwise, cavities, pleural effusion and enlarged lymph nodes are rare. However, SARS had more severe interstitial fibrosis during the absorption phase of pneumonia. After discharge, CT images of SARS patients still showed thickening of the lobular septum, subpleural and distal bronchiolar dilatation, and honeycomb changes [15]. The lung lesions of SARS progressed more rapidly and were termed white out or 'white lung' because consolidation and coalescing infiltrates pervaded the lungs, leaving few recognized air spaces. The COVID-19 patients in this study did not show 'white lung', although we observed lesions involving 15 lung segments in one case, and the distribution of lesions was mainly subpleural. Furthermore, we note that $55 \%$ of cases involved less than three lung segments, which is different from previous reports of adult patients. This finding suggests that COVID-19 has a mild inflammatory infiltration in children, which indicates that they are more likely to recover than adults after symptomatic treatment, and the specific mechanism needs to be further studied.

This study has several limitations. First, the sample size of this study is small because the incidence of children with COVID-19 is not high. Including additional cases could have improved the recognition of image 


\section{WHAT THIS STUDYADDS?}

- Chest computed tomography findings and clinical features of pediatric patients with COVID-19.

features of COVID-19 in children. Second, longitudinal studies on follow-up CT changes during treatment in children need to be carried out. These studies can reflect the course of disease development and pathological changes and may provide valuable experience for future treatment and rehabilitation.

In summary, we found that common chest CT findings in COVID-19 in children include multiple mixed ground glass opacities and consolidation lesions in both lungs, with mostly sub-pleural distribution. The 'crazy-paving sign' was found in a few cases, and the number of lung segments involved was small, with an average of three. More data on this aspect will assist clinicians in diagnosis and management of COVID-19 in children.

Contributors: All authors have contributed, designed and approved the study.

Funding: None; Competing interest: None stated

Ethical approval: Medical Research Ethics Committee of Yichang Central People's Hospital, Yichang, China.

\section{REFERENCES}

1. World Health Organization. Novelcoronavirus-China. Jan12,2020. Available from: http://www.who.int/csr/don/ 12-january-2020-novel-coronavirus-china/en/. Accessed March14, 2020.

2. Chan JF, Yuan S, Kok KH, To KK, Chu H, Yang J, et al. A familial cluster of pneumonia associated with the 2019 novel coronavirus indicating person-to-person transmission: A study of a family cluster. Lancet. 2020; 395: 514-23.

3. Koo HJ, Lim S, Choe J, Choi SH, Sung H, Do KH. Radiographic and CT features of viral pneumonia. Radiographics. 2018;38:719-39.

4. Wu J, Wu X, Zeng W, Guo D, Fang Z, Chen L, et al. Chest CT findings in patients with coronavirus disease 2019 and its relationship with clinical features. Invest Radiol. 2020;10.1097/RLI.0000000000000670. Available form https://journals.lww.com/investigativeradiology/Abstract/ publishahead/Chest_CT_Findings_in_Patients_with_ Corona_Virus.98835.aspx. Accessed March 20, 2020.

5. Ooi GC, Khong PL, Müller NL, Yiu WC, Zhou LJ, Ho JC, et al. Severeacute respiratory syndrome: temporal lung changes at thin-section CT in 30 patients. Radiology. 2004;230: 836-44.

6. Wong KT, Antonio GE, Hui Hui DS, Lee N, Yuen EH, Wu $\mathrm{A}$, et al. Thin-section CT of severe acute respiratory syndrome: evaluation of 73 patients exposed to or with the Disease. Radiology. 2003;228:395-400.

7. Cohen J, Normile D. New SARS-like virus in China triggers alarm. Science. 2020;367: 234-5.

8. Zhu N, Zhang D, Wang W, Li X, Yang B, Song J, et al. A novel coronavirus from patients with pneumonia in China, 2019. N Engl J Med. 2020;382:727-33.

9. Lee N, Hui D, Wu A, Chan P, Cameron P, Joynt GM, et al. A major outbreak of severe acute respiratory syndrome in Hong Kong. N Engl J Med. 2033;348:1986-94.

10. Assiri A, Al-Tawfiq JA, Al-Rabeeah AA, Al-Rabiah FA, Al-Hajjar S, Al-Barrak A, et al. Epidemiological, demographic, and clinical characteristics of 47 cases of Middle East respiratory syndrome coronavirus disease from Saudi Arabia: A descriptive study. Lancet Infect Dis. 2013; 13:752-61.

11. Müller NL, Ooi GC, Khong PL, Zhou LJ, Tsang KW, Nicolaou S. High-resolution CT findings of severe acute respiratory syndrome at presentation and after admission. Am J Roentgenol. 2004;182:9-44.

12. Liu Y, Gayle AA, Wilder-Smith A, Rocklöv J. The reproductive number of COVID-19 is higher compared to SARS coronavirus. J Travel Med. 2020. Available form: https://academic.oup.com/jtm/article/27/2/taaa021/ 5735319. Accessed March 20, 2020.

13. Zhang L, Liu Y. Potential interventions for novel coronavirus in china: A systemic review. J Med Virol. 2020. [Epub ahead of print]. Available form https:// onlinelibrary.wiley.com/doi/full/10.1002/jmv. 25707. Accessed March 20, 2020.

14. Chung M, Bernheim A, Mei X, Zhang N, Huang M, Zeng $\mathrm{X}$, et al. CT imaging features of 2019 novel coronavirus (2019-nCoV).Radiology. 2020. [Epub ahead of print] Available form https://pubs.rsna.org/doi/pdf/10.1148/ radiol.2020200230. Accessed March 20, 2020.

15. Xiangke D, Wanjiang Y, Silun W. Preliminary analysis of clinical images of SARS (in Chinese). Chinese J Radiol. 2003;37:780-3. 